\title{
Flame Spread over a Cellulose Sheet Permeated with an Adhesive
}

\author{
LIJING GAO, RITSU DOBASHI and TOSHISUKE HIRANO \\ Department of Chemical System Engineering \\ The University of Tokyo \\ Tokyo, Japan
}

\begin{abstract}
An experimental study has been performed to cxamine the effects of an adhesive on flame spread phenomena. To characterize these effects, samples having simple structure, i.c., filter paper sheets permeated with two kinds of resins, were utilized for downward flame spread experiments.

It is shown that the assumption of a constant mass burning rate for flame spread over thin solid sheets is not always valid, especially for flame spread over a sample of material permeated with resin. The observed decrease of the mass burning rate with increasing mass per unit arca of polyvinyl acetate resin in the samples cannot be interpreted only in terms of thermal mechanisms. Further, it is pointed out that a mechanism which limits heat transfer to the inside of the gasifying solid is required to explain the observed rapid reduction of the flame spread rate with increasing amounts of permeated urea resin.
\end{abstract}

KEYWORDS: Flame Spread, Solid Combustion, Fire, Adhesivc

\section{INTRODUCTION}

Flame spread over a combustible solid surface is a basic phenomenon in a firc and a number of studies have been performed to characterize the process [1-11]. However, little is known concerning flame spread over a combustible solid which is glued to another solid by an adhesive, although various types of adhesives are widely used in furniture and interior decoration in buildings. The development of a building fire may not be reasonably predicted without an understanding of flame spread over such furniture or interior decoration. 
It is casily imaginable that solid materials glued to each other have complex structures, and that the behavior of a flame spreading over the surface of such material may not be easy to explain in terms of the individual characteristics of the materials and aḍhesive. This is a reason why few studies have examined the role of an adhesive on flame spread over samples of multiple materials joined by adhesive. To obtain a systematic understanding of the effect, it is rcasonable to prepare fuel samples including adhesive, with structures as simple as possiblc $[12,13]$. The direction of flame spread was chosen to be downward because downward flame spread is steady and suitable for examining the basic characteristics of materials in fires. Thus, we have prepared filter paper sheets permeated with an adhesive and have conducted downward flame spread experiments over the sheets.

Two types of adhesives have been used in the present experimental study. One is the main component of urea resin, which is a typical thermosetting resin, and the other is polyvinyl acctatc resin, which is a typical thermoplastic resin. Both are widely used in furniturc manufacture and interior decoration of buildings. Examining the effects of each of these adhesives on flame spread as well as the differences between them provides us necessary knowledge for evaluating fire hazards for combined materials.

\section{EXPERIMENTAL}

The filter paper sheets used in the present experimental study are of $10 \mathrm{~cm} \times 20 \mathrm{~cm}$ in surface area and $0.53 \mathrm{~mm}$ in thickness. They were prepared by cutting papers of a large size and uniform quality. The mass per unit area of the paper sheet was $14 \mathrm{mg} / \mathrm{cm}^{2}$.

The mass percentage of urea resin in urea adhesive used for the present experiments is $45.7 \%$ in water and that of polyvinyl acetate adhesive is $41.4 \%$ in water. Each of these is diluted with water to make solutions having desired compositions.

Each filter paper sheet was placed in a solution of a certain concentration of an adhesive for 3 minutes. Then the sheet was removed from the solution and placed in a drying oven. After drying in the oven at $105 \pm 2{ }^{\circ} \mathrm{C}$ for three hours, it was kept in a desiccator until the start of preparation for a test. Through this process, we could prepare samples of dry filter paper uniformly permeated with urea resin or polyvinyl acetate resin in the range of () to 30 $\mathrm{mg} / \mathrm{cm}^{2}$. The density $\rho$ of samples are $0.264 \mathrm{~g} / \mathrm{cm}^{3}$ for dried pure filter paper and from 0.264 to 0.830 $\mathrm{g} / \mathrm{cm}^{3}$ for filter paper sheets permeated with urea resin or polyvinyl acetate resin .

Just before each test, a test sample was taken from the desiccator and set in a pair of vertical aluminum frames as shown in Fig. 1. Since both sides of the

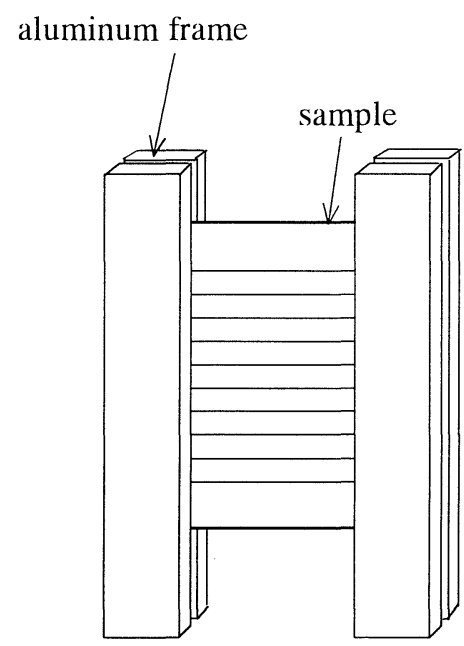

FIGURE 1 Experimental setup. 
sample were held by the frames, the exposed for burning during the test area was reduced to 5 $\mathrm{cm} \times 20 \mathrm{~cm}$.

A slit burner was used to uniformly ignite the sheet on its top edge, and downward flame spread phenomena were recorded using a video camera and then analyzed. In the analysis, the flame spread rate was examined by measuring the times when the leading flame edge passed lines printed on the sample surface parallel to its shorter side at intervals of $10 \mathrm{~cm}$ [3].

\section{RESULTS AND DISCUSSION}

\section{Influence of the Process Used to Prepare Test Samples}

In the present study, test samples were processed in our laboratory. The effects of the process used to permeate a sample with resin on flame spread phenomena should be minimized or at least known, and reproducibility of an indicated quality for each paper should be maintained. Thus, before examining the effects of adhesives, the effects of the process on flame spread phenomena were examined $[12,13]$.

Three test samples of filter paper are prepared. The first one is a filter paper left in a room for a few days, the second one is dried in the oven and kept in a desiccator, and the third one is dried after being permeated with pure water.

No apparent difference could be found for the flame spread over these three different samples. The shape and color of the flames were the same, and the flame spread rate for each sample was constant throughout the test. The only difference noted was for the flame spread rates. The measured flame spread rates were $0.137,0.148$, and $0.148 \mathrm{~cm} / \mathrm{s}$ for the samples left in a room for a few days, dried in the oven, and dried after permeation with pure water, respectively. The fact that the first one has a slightly smaller flame spread rate than the latter two is easily understood by considering the effect of moisture on the flame spread rate as pointed out in previous studies [3,14]. These results indicate that the process used for sample permeation does not itself change the flame spread behavior of filter paper.

\section{Behavior of Flames and Flame Spread Rates}

Flame spread phenomena depend strongly on the kind and amount of the adhesive permeated into the sample. Figure 2 shows the behavior of flames spreading over 4 test samples having different compositions of added resin plotted as $\mathrm{x}-\mathrm{t}$, distance-time diagrams.

When a flame spreads downward over a sample permeated with $4 \mathrm{mg} / \mathrm{cm}^{2}$ of polyvinyl acetate resin, the flame spread rate is almost constant throughout the test. The value of the flame spread rate is smaller than that over a sample of pure filter paper. The former is about $73 \%$ of the latter. In this case, the appearances of the spreading flame are similar to that for flame spread over a test sample of pure filter paper, while the shape and dimension of the pyrolysis zone, carbonized residue and ash behind the leading flame edge appear to be different. For 
flame spread over the test sample permeated with polyvinyl acetate resin the pyrolysis zone cannot be casily distinguished from the carbonized residuc which is much wider in length than observed for flame spread over the pure filter paper.

The reduction of the flame spread rate is much morc marked in the case where urca resin is permeated into the filter paper sheet compared to that for which polyvinyl acetate resin is used. This is easily confirmed by Fig. 2. It is seen that the flame spread rate over a sample permeated with $4 \mathrm{mg} / \mathrm{cm}^{2}$ of urea resin is about $53 \%$ of that for purc filter paper. A further increase of the amount of permeated urea resin results in interruption of flame spread. In the typical example presented in Fig. 2 for a sample permeated with $5 \mathrm{mg} / \mathrm{cm}^{2}$ of urca resin, flame spread stops at a point less than $2.0 \mathrm{~cm}$ from the top where the sample was ignited. The behavior of the sample during flame spread in this case is very different from that in the casc of flame spread over filter paper without added urea. Behind the leading flame cdge, the shect is carbonized and remains as a carbon residuc. The flame becomes smaller as the amount of permeated urea resin increases, and ceases for amounts of permeated urea resin larger than 4 $\mathrm{mg} / \mathrm{cm}^{2}$, the flame decreases in size continuously during flame spread until it vanishes.

The gradient of a line representing flame spread on an $x-t$ diagram such as show'n in Fig. 2 corresponds to the flame spread rate. Figure 3 shows the results of flame spread rate measurements for samples permeated with various amounts of adhesives. In the figure the flame spread rate $V$ is plotted against the mass $\delta \rho_{r}$ of permeated adhesive per unit area. $\rho_{r}$ is the density of an adhesive in the sample and $\delta$ is the sample thickness. It is secn that the flame spread rate decreases with increasing amounts of both adhesives. The flame spread ratc over a sample permeated with about $30 \mathrm{mg} / \mathrm{cm}^{2}$ of polyvinyl acctatc resin, the maximum permeated amount, is about $1 / 5$ of that for filter paper without adhesive. It was confirmed that the flame continues to spread even if the sample consists of pure polyvinyl acetate resin. These results indicate that the permeation with polyvinyl acetate resin reduces the flame spread ratc, but is not effective in stopping the spread.

The flame spread rate over samples permeated with urea resin decreases more markedly with increasing amounts of urea resin than for polyvinyl acetate resin. Further, the flame no longer spreads if the permeated amount of urea resin per unit area is more than $4 \mathrm{mg} / \mathrm{cm}^{2}$, which is about $1 / 4$ of the mass of the filter paper. These results indicate that urea resin is cffective in preventing and interrupting flame spread.

When samples permeated with more than $4 \mathrm{mg} / \mathrm{cm}^{2}$ of urea resin arc ignited, a flame is established and starts to spread. As mentioned above, the flame stops after spreading for a short distance. In this case, the distance from the line of ignition to that for cessation of flame spread depends on the amount of permeated urea resin. Figure 4 shows the results of measurement of the distance as a function of $\delta \rho_{r}$. It is seen that the distance rapidly decreases with increase in the amount of permeated urea resin. For samples permeated with more than $10 \mathrm{mg} / \mathrm{cm}^{2}$ of urea resin, the flame stops spreading just after ignition.

\section{Controlling Mechanisms of Flame Spread}

In a discussion on flame spread mechanisms, it is important to explore the process of heat 


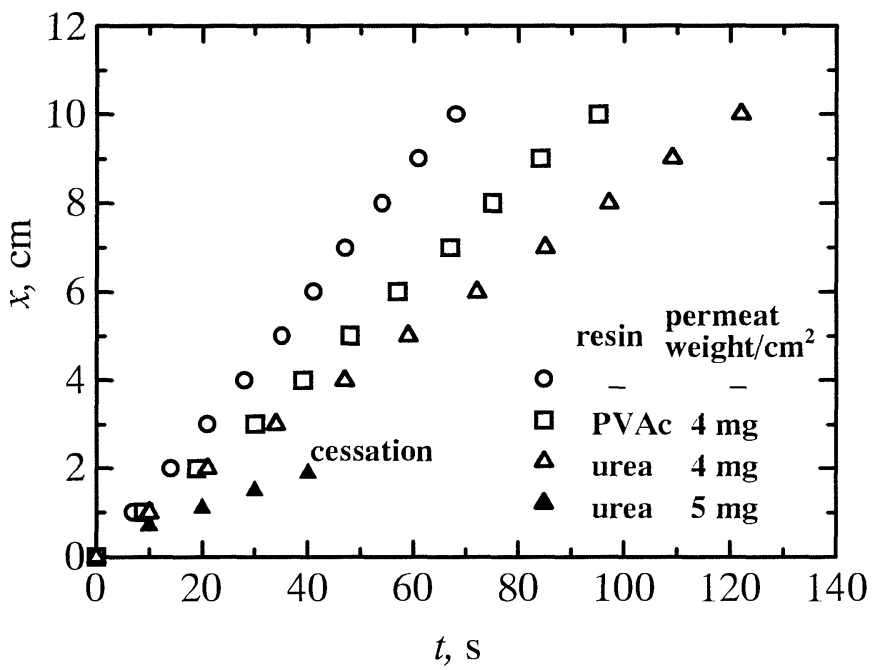

FIGURE $2 x$-t, distance-time diagrams representing flame spreading over 4 samples.

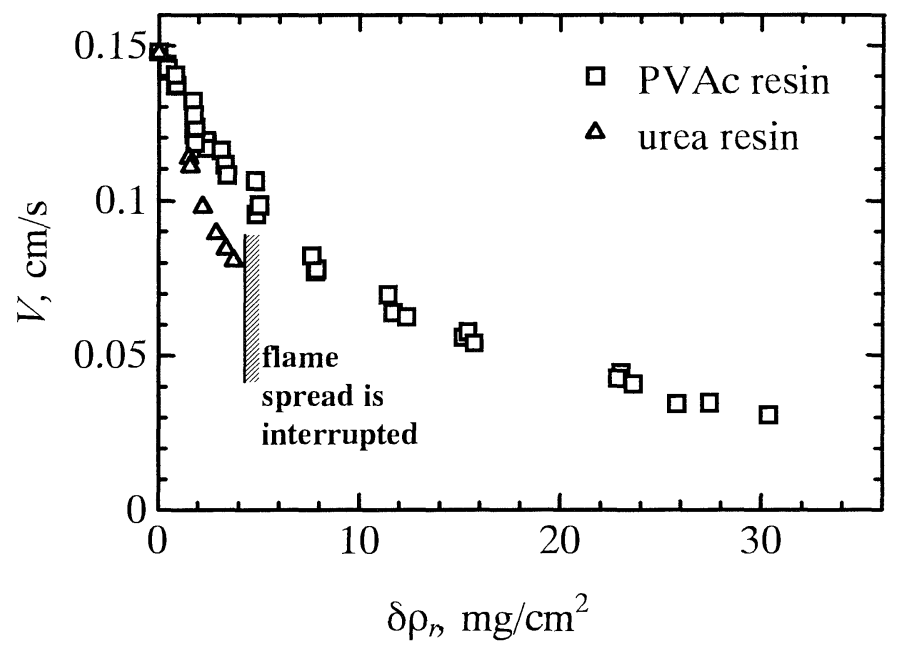

FIGURE 3 Flame spread rates for samples permeated with adhesives. $\quad V$ : flame spread rate, $\delta$ : sample thickness, $\rho_{r}$ : adhesive mass permeated per unit area. 
transfer to the not-as-yet burning part of the sample. In previous studies on flame spread, various equations have been derived to predict the flame spread rate on the basis of heat transfer considerations. The following is one such relation [4].

$$
\begin{aligned}
V \delta \rho=\frac{\delta}{c \int_{-\delta / 2}^{\delta / 2}\left(T_{x=0}-T_{r}\right) \mathrm{d} y}\{ & 2 \int_{-\infty}^{0}\left(\lambda_{g} \frac{\partial T}{\partial y}\right)_{w} \mathrm{~d} x \\
& \left.-2 \int_{-\infty}^{0} \varepsilon \sigma\left(T_{w}{ }^{4}-T_{r}{ }^{4}\right) \mathrm{d} x+\int_{-\delta / 2}^{\delta / 2}\left(\lambda_{s} \frac{\partial T}{\partial x}\right)_{x=0} \mathrm{~d} y\right\},
\end{aligned}
$$

where $\rho$ and $c$ are the density and specific heat of the sample, respectively. $T$ is temperature and $\lambda_{g}$ and $\lambda_{s}$ are thermal conductivities of the gas and solid, respectively. The coordinates are shown in Fig. 5, and the suffixes, $x=0, r$, and $w$ for $T$ refer to the leading edge of the pyrolysis zone, room, and sample surface, respectively.

In the present case, the thickness of the sample is small, and the flame is not so bright, so that the second and third terms in the braces have been neglected as in previous studies $[3,7,8]$. Also, the first term in the braces and the denominator of the term in front of the braces have been assumed to be constant. If such assumptions are reasonable, $V \delta \rho$ should be constant or very close to constant. Thus, values of $V \delta \rho$ were examined. The results are shown in Fig. 6

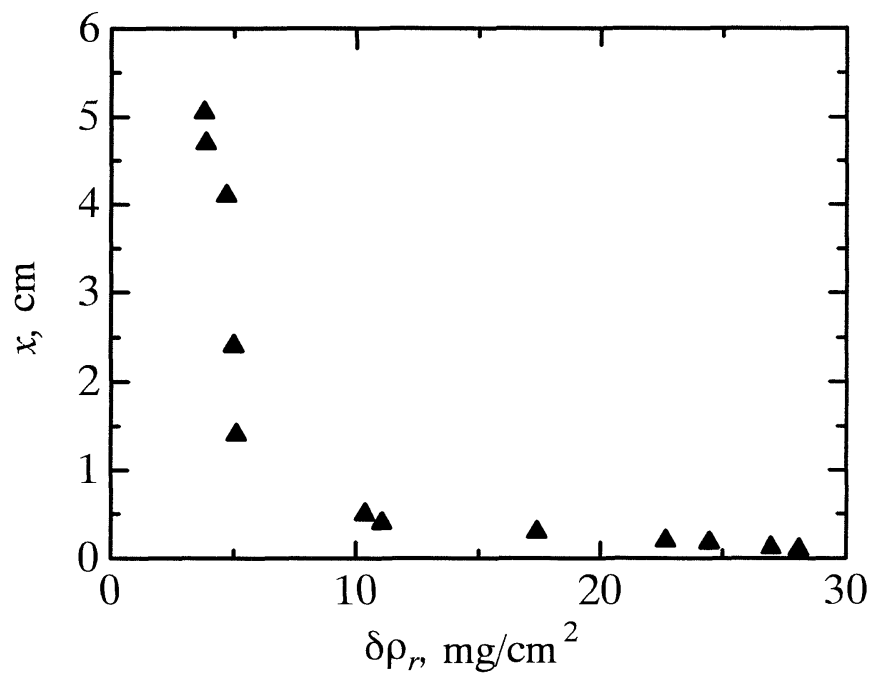

FIGURE 4 Distance from the line of ignition to that of cessation of flame spread over samples permeated with urea resin. 


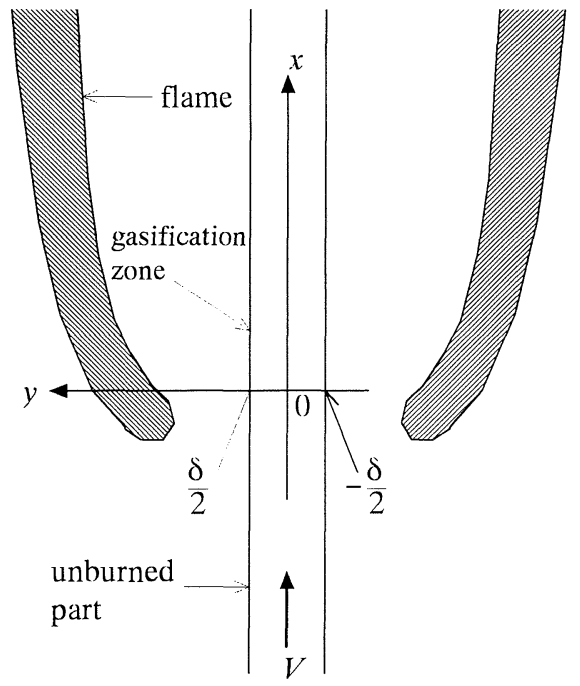

FIGURE 5 Coordinates for discussion on flame spread over thin solid.

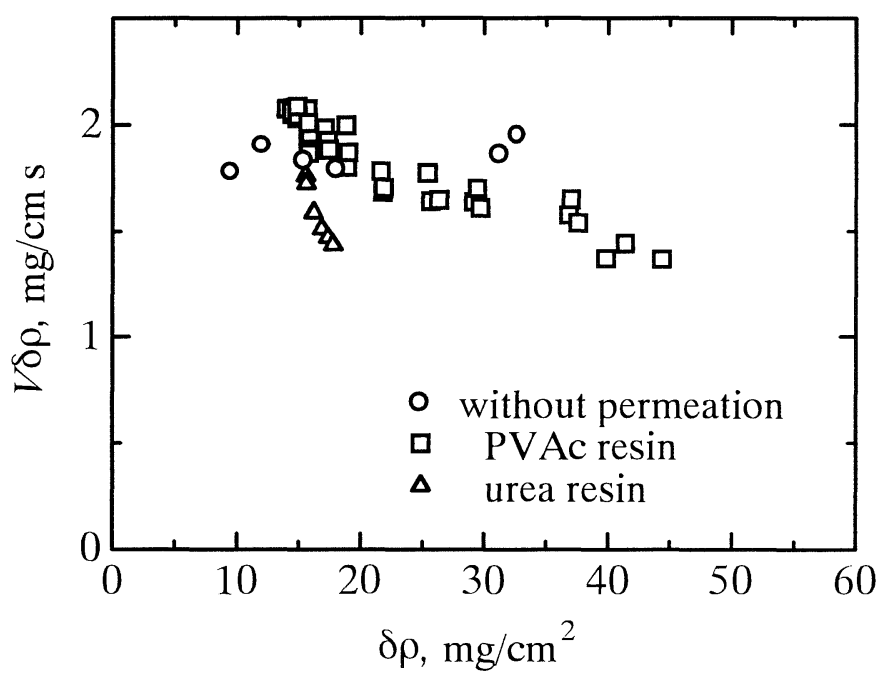

FIGURE 6 Variation of mass burning rate $V \delta \rho$ with mass $\delta \rho$ per unit area of samples with or without permeated adhesive. 
where $V \delta \rho$ is plotted against $\delta \rho$. In the figure, data for samples of filter paper sheets of different thicknesses obtained in the previous studies of Campbell [15] and Hirano [16] are also presented.

It is seen that for flame spread over filter paper without permeation the value of $V \delta \rho$ is almost constant, while for those with adhesive permeation the value decreases with the increase of $\delta \rho$. Especially, in the case for flame spread over a sample permeated with urea resin, the decrease of flame spread rate is marked with the increase in the amount of permeated urea resin. This result indicates that the assumption of constant $V \delta \rho$ is not always valid especially for the flame spread over a sample of material permeated with resin.

As mentioned previously, even if a sample is of pure polyvinyl acetate resin, a flame does not stop spreading over it, i.e., the permeation of polyvinyl acetate resin is not effective in halting flame spread. Indeed, the appearance of a flame spreading over a sample permeated with polyvinyl acetate resin is almost independent of the amount of permeated polyvinyl acetate resin. If we restrict our discussion to interpretation of flame spread solely on the basis of heat transfer, there are two possible reasons for decreasing $V \delta \rho$ with increasing $\delta \rho$. One is the decrease of the first term in the braces and the other is the increase of the denominator of the term in front of the braces in Eq. (1). The former can be a main reason when the heat transfer from the flame to the solid increases with increasing the amount of the permeation resin. If the heat transfer from the flame to the solid can be assumed to be almost constant, the latter should be a main reason. Since specific heats of paper and both resins are almost the same, $T_{x=0}$ should increase with increasing $\delta \rho$. However, this is not logical. The temperature $T_{x=0}$ needed to start gasification of the filter paper permeated with polyvinyl acetate resin necessarily decreases with increasing the amount of the permeation resin because the pyrolysis temperature of filter paper is higher than the depolymerization temperature of the polyvinyl acetate under the conditions of flame spread in the present experimental study. Thus, the decrease of $V \delta \rho$ with the increase of $\delta \rho$ cannot be interpreted simply by the increase of the denominator of the term in front of the braces in Eq.(1). The composition of the combustible gas ejected from the surface of the pyrolysis-depolymerization region should be taken into account for interpreting the decrease of $V \delta \rho$ with the increase of $\delta \rho$ in the case of the flame spread over a paper sheet permeated with polyvinyl acetate resin.

The rapid reduction of the flame spread rate with increasing amount of permeated urca resin may not be interpreted only by considering the composition of the combustible gas ejected from gasifying zone beneath the leading flame edge. The increase of the amount of permeated urea resin results in the cessation of flame spread. This implies that the urea resin is effective in retarding flame spread. In this case the flame size decreases with increasing the amount of the permeated urea resin. The decrease of the flame size is in general attributable to the reduction of the gasification rate caused by blockage of heat transfer to the gasifying part. A possible mechanism of this heat blockage is due to carbonization of the sample surface. A carbon layer can be a barrier which reduces the rate of heat transfer. Urea resin is effective in establishing such a carbon layer covering the gasifying surface. 


\section{CONCLUSIONS}

An experimental study has been performed to examine the effect of adhesives on flame spread phenomena. To develop a systematic understanding of the effect, samples of a simple structure, i.e., filter paper sheets permeated with an adhesive of polyvinyl acetate resin or urea resin were adopted for experiments. Major conclusions derived through the present study are as follows:

1. The appearance of a flame spreading over a sample permeated with polyvinyl acetate resin is similar to that over a sample without added resin, but the flame spread rate decreases with increasing amount of permeated polyvinyl acetate resin.

2. The reduction of the flame spread rate is much larger in the case when urca resin is permeated into the filter paper sheet compared to that in the case when polyvinyl acetate resin is permeated. A further increase of the amount of permeated urea resin results in cessation of flame spread, and the distance from the line of ignition to that of where the flame halts decreases with increasing the amount of permeated urea resin.

3. The assumption of constant mass burning rate is not always valid especially for flame spread over a sample of material permeated with resin. The mass burning rate decreases with increasing mass per unit area. The composition of combustible gases ejected from surface of a pyrolysis-depolymerization region should be taken into account when interpreting the decrease of mass burning rate with the increase of mass per unit area in the case of the flame spread over a sample permeated with polyvinyl acetate resin.

4. The rapid reduction of the flame spread rate over a sample with increasing amount of permeated urea resin may not be interpreted even by considering the composition of the combustible gas ejected from gasifying zone beneath the leading flame edge. A possible mechanism for the heat blockage, which would cause the reduction of flame spread rate or cessation of flame spread, is the possible carbonization of the sample surface. A carbon layer can be a barrier which reduces the rate of heat transfer to the solid. Urea resin is effective in establishing such a carbon layer covering the gasifying part.

\section{ACKNOWLEDGEMENT}

The present study is partly supported by Japan Fire Retardant Association through the Japan Society for Safety Engineering. The authors are most grateful to Dr. Masataro Suzuki for his stimulating discussion and suggestions during the present study.

\section{REFERENCES}

1. Friedman, R., "A Survey of Knowledge About Idealized Fire Spread Over Surfaces,” Fire Research Abstracts and Reviews, 10, 1-8, 1968.

2. Emmons, H. W., and Shen, T., "Fire Spread in Paper Arrays," 13th Symposium 
(International) on Combustion, pp.917-926. The Combustion Institutc, Pittsburgh, Pa., 1971.

3. Hirano, T., Noreikis, S. E., and Waterman, T. E., "Postulations of Flamc Spread Mechanisms," Combustion and Flame, 22, 353-363, 1974.

4. Hirano, T., and Tazawa, K., "Effect of Thickness on Downward Flame Spread Over Paper,” Bul. Japanese Assoc. Fire Science and Engineering, 26:1, 7-13, 1976.

5. Hirano, T., Sato, K., and Tazawa, K., "Instability of Downward Flame Spread Over Paper in an Air Stream," Combustion and Flame, 26, 191-200, 1976.

6. Tazawa, K., and Hirano, T., "Upward Flame Spread Over Paper in an Air Stream," Bul. Japanese Assoc. Fire Science and Engineering, 27:1, 9-16, 1977.

7. Fernandez-Pello, A. C., Ray, S. R., and Glassman, I., "Downward Flame Spread in an Opposed Forced Flow," Combustion Science and Technology, 19, 19-30, 1978.

8. Hirano, T., Koshida, T., and Akita, K., "Flame Spread Mechanisms Over PMMA Surfaces," Bul. Japanese Assoc. Fire Science and Engineering, 27:2, 33-39, 1977.

9. Fernandez-Pello, A. C., Hirano, T., "Controlling Mechanisms of Flamc Spread," Combustion Science and Technology, 32, 1-31, 1983.

10. Sato, K., Miki, K., and Hirano, T., " Flame Spread Over Paper in an Air Stream With a Velocity Change,” J. Heat Transfer, 106, 7(07-712, 1984.

11. Suzuki, M., Dobashi, R., and Hirano, T., "Behavior of Fires Spreading Downward Over Thick Paper," 25th Symposium (International) on Combustion, pp.1439-1446. The Combustion Institute, Pittsburgh, Pa., 1994.

12. Gao, L., Dobashi, R., and Hirano, T., "A Study of Effects of an Adhesive on Flame Spread Across a Paper Sheet Surface," Proceedings of Annual Meeting of Japan Association for Fire Science and Engineering, pp.108-111, 1995.

13. Gao, L., A Study of Effects of Adhesives on Flame Spread Over The Surface of Combustible Solid Materials, Master's Thesis, Univ. Tokyo, Tokyo, 1996.

14. Izumikawa, M, Mitani, T, and Niioka, T., "Flame Spread of Paper Sheets Containing Suppressants” Bul. of JSME, 52:475 (Ser.B), 1413-1419, 1986.

15. Campbell, A, S., "Some Burning Characteristics of Filter Paper," Combustion Science and Technology, 3, 103-120, 1971.

16. Hirano, T., "Mechanisms of Flame Spread Over Paper Sheets;" Nensho-Kenkyu, 36, 1-33, 1974. 